\title{
Factor V Leiden 1691G > A mutation and the risk of recurrent pregnancy loss (RPL): systematic review and meta-analysis
}

\author{
Mohammad Masoud Eslami ${ }^{1}$, Majid khaliiii, ${ }^{2,3}$, Mina Soufizomorrod', Saeid Abroun ${ }^{1}$ and Bahman Razi ${ }^{* *}$
}

\begin{abstract}
Background: Although numerous replication case-control studies have attempted to determine the association between Factor V Leiden (FVL) 1691G > A mutation and susceptibility to Recurrent pregnancy loss (RPL), there have been confliction among the results of various ethnic groups. To address this limitation, here we implemented first meta-analysis to provide with consistent conclusion of the association between FVL 1691G > A mutation and RPL risk.

Methods: After a systematic literature search, pooled odds ratio (OR) and their corresponding $95 \%$ confidence interval (Cl) were used to evaluate the strength of the association. Additionally, meta-regression analyses were performed to find potential source of heterogeneity.

Results: In this meta-analysis, 62 studies, containing 10,410 cases and 9406 controls, were included in quantitative analysis. Overall population analysis revealed a significant positive association in the dominant $(\mathrm{OR}=2.15,95 \% \mathrm{Cl}=$ 1.84-2.50, $P<0.001)$, over-dominant $(\mathrm{OR}=1.88,95 \% \mathrm{Cl}=1.61-2.19, P<0.001)$, allelic $(\mathrm{OR}=2.05,95 \% \mathrm{Cl}=1.79-2.35$, $P<0.001)$, and heterozygote $(\mathrm{OR}=1.97,95 \% \mathrm{Cl}=1.68-2.30, P<0.001)$ models. Moreover, a significant association of dominant $(\mathrm{OR}=3.04,95 \% \mathrm{Cl}=2.04-4.54, P<0.001)$, over-dominant $(\mathrm{OR}=2.65,95 \% \mathrm{Cl}=1.74-4.05, P<0.001)$, and heterozygote $(\mathrm{OR}=2.67,95 \% \mathrm{Cl}=1.81-4.22, P<0.001)$ models was found in the Iranian population. The subgroup analysis indicated strong significant association in Asian, European, Africa population, and case-control studies but not in South Americans and cohort studies.
\end{abstract}

Conclusion: The FVL 1691G > A mutation and the risk of RPL confers a genetic contributing factor in increasing the risk of RPL, particularly in Iranians, except for South Americans.

Keywords: Recurrent pregnancy loss, Factor V Leiden, 1691G > A mutation, Meta-analysis, Meta-regression

\section{Introduction}

Recurrent pregnancy loss (RPL) is a heterogeneous disorder which affects women of reproductive age. Recently, The American Society of Reproductive Medicine has defined RPL as two or more than two failed pregnancies before the 20th week of pregnancy [1-3]. Overall, $1-5 \%$ of women during reproductive ages could be affected [4].

\footnotetext{
* Correspondence: b.razi@modares.ac.ir

'Department of Hematology, Faculty of Medical Sciences, Tarbiat Modares University, North Kargar Av, Tehran 14117, Iran

Full list of author information is available at the end of the article
}

From pathophysiological point of view, RLP might be influenced by various items, such as genetic factors (chromosomal aberrations, genetic polymorphisms), infectious diseases, structural abnormalities of the uterus, coagulative disorders (thrombophilia), endocrinological problems (thyroid disease and diabetes), and immunological disease (autoimmune disorder and inflammatory diseases) [5-7]. With considering these factors, still approximately 40 to $50 \%$ of cases remained idiopathic [8].

Although pregnancy as a physiological condition is associated with a hypercoagulable state, and the contact 
between placenta and maternal circulation is crucial for the establishment of a successful pregnancy, but any abnormality in this circulation, especially abnormal blood clotting in the small placental blood vessels, may results in RPL $[9,10]$. During last decades, thrombophilia attracted a lot of attention as a risk factor for RLP. Thrombophilia is characterized as a hemostatic disorder which leads to an increased tendency of thromboembolic processes. Classically, thrombophilia could be classified into acquired and inherited forms $[11,12]$. In this regards, antiphospholipid syndrome is an established acquired thrombophilia factor which increase the risk of RPL. Among inherited factors, mutation in Factor V Leiden (FVL) of the FV gene, G20210A of the FII (prothrombin) gene, and C677T of the methylenetetrahydrofolate reductase (MTHFR) gene are believed to play a key role in pathogenesis of RPL [13, 14].

FVL mutation shows an autosomal dominant pattern which occurs by substitution of guanine by adenine (CGA--- > CAA) at the nucleotide 1691 in the exon 10. As a result of this missense mutation, arginine (Arg) at amino acid 506 is substituted with glutamine (Gln), leading to generation of FVL resistant to the activated protein $\mathrm{C}$ (APC). APC is a natural anticoagulant which in normal situation cleaves activated factor $\mathrm{V}$ at amino acid 506 and makes it inactive [15-20].

Studies have shown that FVL mutation increases the risk of venous thrombosis 7 times in heterozygote and 80 times in homozygote carriers. In addition, it has been reported that this mutation increases the risk of preeclampsia in FVL carriers [21, 22]. The exact mechanism that FVL mutation influence the etiology of RPL is a controversial issue and has not yet been divulged thoroughly, but several studies suggested that production of micro thrombosis could sediment in delicate placental blood vessels and cause placental infarction and subsequent maternal and fetal complications [23, 24].

In spite of all findings, still the exact association between FVL mutation and the risk RPL is unclear and several investigators worldwide try to clarify this question. Therefore, here we conducted the first and the most comprehensive meta-analysis on the association between FVL 1691G > A mutation and risk of RPL by exerting 62 studies encompassing 10,410 cases and 9406 health control to achieve more reliable conclusion.

\section{Methods}

Ethical approval is not necessary for this meta-analysis. The current meta-analysis was conducted according to the Preferred Reporting Items for Systematic reviews and Meta-Analyses (PRISMA) statement [25], including publication search, study selection, inclusion and exclusion criteria, data extraction, quality assessment, and statistical analysis.

\section{Publication search}

A comprehensive systematic search in the ISI Web of Science, Scopus, and PubMed/Medline databases was conducted to retrieve all publications evaluating the associations between FVL 1691G > A mutation and susceptibility to RPL prior to May 2020. The following combinations of key words were used: ("Miscarriage" OR "abortion" OR "pregnancy loss" OR "habitual abortion" OR "fetal loss" OR "Recurrent Pregnancy Loss") AND ("Factor V Leiden" OR "FV Leiden" OR "1691G > A" OR "rs6025") AND ("polymorphism"” OR "variant" OR "mutation" OR "genotype" OR "allele" OR "single nucleotide polymorphism" OR "SNP"). In spite of detailed search, a manual cross-check of eligible studies and reviews was carried out to include other potential studies. Original data in English language and human population studies were collected.

\section{Study selection}

Primary search strategy generates 1266 studies that were exported into Endnote X8 software. The duplicated studies were removed and title \& abstract of remaining studies were reviewed by two investigators and irrelevant studies were excluded. Full-text verification was performed if we could not classify studies based on title \& abstract. Any disagreements during study selection were discussed and resolved by consensus.

\section{Inclusion and exclusion criteria}

Studies considered eligible if they met the following inclusion criteria: a) Studies concerning the association between FVL 1691G > A mutation and susceptibility to recurrent pregnancy loss as the main outcome; b) Studies that their case group have recurrent pregnancy loss (two or more times of abortion); c) Studies with case-control and cohort design; d) Studies reporting sufficient data of genotype or allele frequency that could confer feasibility of calculating the odds ratios (ORs) and 95\% confidence intervals (CIs). On the other hand, duplicates, case reports, book chapters, reviews, letter to editor, studies with insufficient data, and abstracts were all excluded.

\section{Data extraction and quality assessment}

According to a standardized extraction form, the following data were independently extracted by two investigators: the first author's last name, journal and year of publication, country of origin, ethnicity, allele and genotype frequency in cases and controls, mean or range of age, genotyping method, and total sample size of cases and controls. The third investigator finalized the extracted data, and potential discrepancies were resolved by consensus. For quality assessment of the included publications, the Newcastle-Ottawa Scale (NOS) was applied [26]. In this respect, studies with $0-3,4-6$ or 7-9 scores were of, respectively, low, moderate, and high-quality. 


\section{Statistical analysis}

Deviation from Hardy-Weinberg equilibrium (HWE) for distribution of the genotype frequencies was analyzed by $x^{2}$-test in the control group. The strength of the association between FVL 1691G > A mutation and RPL risk was evaluated by the pooled OR and its corresponding 95\% CI. Different comparison models for FVL 1691G > A mutation were as follow: dominant model (AA+GA vs. GG), overdominant model (GA vs. GG + AA), allelic model (A vs. G), and heterozygote (GA vs. GG). It should be noted that due to the AA genotype frequency of zero in both cases and controls, the recessive and homozygote models were not calculable. Presence of heterogeneity between included studies was estimated by Cochran's Q-statistic ( $P$ value $<$ 0.10 was considered as statistically significant) [27]. Besides, to report quantitative heterogeneity I-squared $\left(\mathrm{I}^{2}\right)$ tests was used. The fixed-effected model (FEM) was used if $\mathrm{P}_{\mathrm{Q} \text {-statis- }}$ tic $>0.10$ or $\mathrm{I}^{2}$ was $<50 \%$; otherwise, the random-effected model (REM) was applied. In order to assessed the predefined sources of heterogeneity among included studies, subgroup analysis and meta-regression analysis based on year of population, the continent of the study population, and genotyping method were performed. Additionally, sensitivity analysis was conducted in presence of heterogeneity [28, 29]. Publication bias was estimated by Begg's funnel plots and Egger's regression test $(P$ value $<0.05$ was considered as statistically significant) [30,31]. The funnel plot asymmetry was assessed with the Egger's test. Practically, in case of no evidence of publication bias, studies with high precision (large study effects) will be located near the average line, and studies with low precision (small study effects) will be spread equally on both sides of the average line; any deviation from this shape can indicate publication bias. The data analyses were carried out using STATA (version 14.0; Stata Corporation, College Station, TX) and SPSS (version 23.0; SPSS, Inc. Chicago, IL) software.

\section{Results}

\section{Study characteristics}

The four-phase search and screening process of the literatures based on the PRISMA statement is depicted in the Fig. 1. According to the aforementioned keywords, a

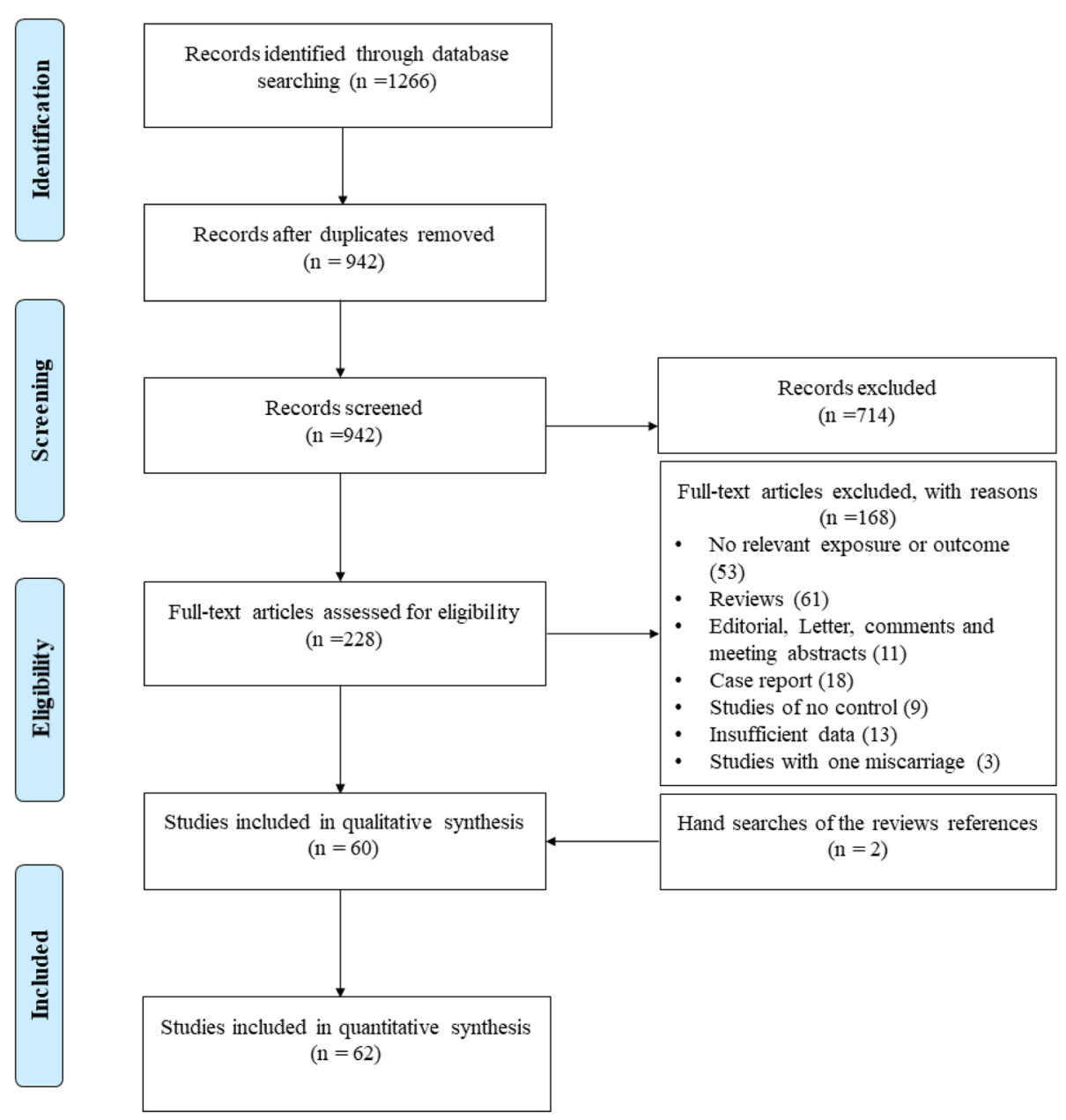

Fig. 1 Flow diagram of study selection process 
Table 1 Characteristics of studies included in meta-analysis

\begin{tabular}{|c|c|c|c|c|c|c|c|c|}
\hline Study author & Year & Country & $\begin{array}{l}\text { Study } \\
\text { design }\end{array}$ & Ethnicity & $\begin{array}{l}\text { Total cases/ } \\
\text { controls }\end{array}$ & $\begin{array}{l}\text { Age } \\
\text { case/control (Mean) }\end{array}$ & $\begin{array}{l}\text { Genotyping } \\
\text { method }\end{array}$ & $\begin{array}{l}\text { Quality } \\
\text { score }\end{array}$ \\
\hline Souza et al. [34] & 1999 & Brazil & case-control & South America & $56 / 384$ & $29.6 / 24.3$ & RLFP-PCR & 7 \\
\hline Brenner et al. [35] & 1999 & Israel & case-control & Asia & $76 / 106$ & $31 \pm 5 / 31 \pm 6$ & RLFP-PCR & 6 \\
\hline Wramsby et al. [36] & 2000 & Sweden & case-control & Europe & $62 / 69$ & $21-39 / 21-39$ & RLFP-PCR & 7 \\
\hline Murphy et al. [37] & 2000 & Ireland & case-control & Europe & $41 / 540$ & $32 \pm 0.74 / N R$ & RLFP-PCR & 6 \\
\hline Pihusch et al. [33] & 2000 & Germany & case-control & Europe & $102 / 128$ & $35 / 32$ & RLFP-PCR & 6 \\
\hline Younis et al. & 2000 & Israel & case-control & Asia & 78/139 & $30.0 \pm 4.4 / 30.7 \pm 4.2$ & RLFP-PCR & 6 \\
\hline Foka et al. [14] & 2000 & Greece & case-control & Europe & $80 / 100$ & $33 / 35$ & RLFP-PCR & 6 \\
\hline Rai et al. & 2001 & London & cohort & Europe & $1111 / 150$ & $33.5 / 33$ & RLFP-PCR & 8 \\
\hline Carp et al. & 2002 & Israel & case-control & Asia & $108 / 82$ & $31 / 36$ & RLFP-PCR & 6 \\
\hline Finan et al. [38] & 2002 & Lebanon & case-control & Asia & $110 / 67$ & $32.3 \pm 5.3 / 33.9 \pm 7.3$ & RLFP-PCR & 6 \\
\hline Hohlagschwandtner et al. & 2003 & Australia & case-control & Oceania & $145 / 101$ & $32 / 56$ & Multiplex PCR & 7 \\
\hline Pauer et al. [39] & 2003 & German & case-control & Europe & $30 / 122$ & $31.3 / \mathrm{NR}$ & RLFP-PCR & 6 \\
\hline Mtiraoui et al & 2004 & Tunisia & case-control & Africa & $146 / 99$ & $29.0 \pm 6.1 / 28.9 \pm 5.3$ & RLFP-PCR & 6 \\
\hline Aksoy et al. & 2005 & Turkey & case-control & Europe & $41 / 50$ & $32 \pm 5.54 / 29 \pm 4.66$ & PCR & 5 \\
\hline Mahjoub et al. [40] & 2005 & Tunisia & case-control & Africa & $200 / 200$ & $28.68 \pm 5.61 / 28.24 \pm 5.51$ & RLFP-PCR & 8 \\
\hline Ulukus et al. & 2006 & Turkey & case-control & Europe & $10 / 53$ & $29.1 \pm 5.2 / 28.0 \pm 4.8$ & PCR & 5 \\
\hline Sotiriadis et al. & 2006 & Greece & case-control & Europe & $99 / 102$ & $32.2 / 32.2$ & RLFP-PCR & 6 \\
\hline Mohammad et al. [21] & 2007 & Syrian & case-control & Asia & $35 / 45$ & $29.6 \pm 6.3 / 28.8 \pm 6.8$ & $Q-P C R$ & 5 \\
\hline Altintas et al. [41] & 2007 & Turkey & case-control & Europe & $114 / 185$ & $30.6 \pm 4.4 / 30.5 \pm 4.3$ & $Q-P C R$ & 7 \\
\hline Toth et al. [42] & 2008 & Germany & case-control & Europe & $151 / 157$ & $33.2 \pm 4.6 / 45.2 \pm 12.6$ & RLFP-PCR & 7 \\
\hline Pasquier et al & 2008 & France & case-control & Europe & $311 / 599$ & $32.8 / 34.3$ & Q-PCR & 8 \\
\hline Biswas et al. [43] & 2008 & India & case-control & Asia & $85 / 31$ & $27.9 \pm 0.3 / 26 \pm 0.5$ & RLFP-PCR & 6 \\
\hline Lvanov et al. & 2009 & Bulgaria & case-control & Europe & $153 / 100$ & $29.7 / 31.0$ & RLFP-PCR & 7 \\
\hline Mukhopadhyay et al. [44] & 2009 & India & case-control & Asia & $84 / 80$ & $24.9 \pm 3.3 / 24.9 \pm 3.3$ & RLFP-PCR & 6 \\
\hline Ciacci et al. [45] & 2009 & Italy & case-control & Europe & $39 / 72$ & $36.24 \pm 8.26 / 30.10 \pm 8.60$ & Multiplex PCR & 6 \\
\hline Mohamed et al. [46] & 2010 & Egypt & case-control & Africa & $20 / 20$ & $29.0 \pm 4.80 / 31.4 \pm 6.82$ & $P C R$ & 5 \\
\hline Hussein et al. [47] & 2010 & Palestine & case-control & Asia & $145 / 205$ & $31.9 / 32$ & ARMS-PCR & 7 \\
\hline Serrano et al. [17] & 2011 & Portugal & case-control & Europe & $100 / 100$ & $32 \pm 4.25 / 30.9 \pm 5.19$ & PCR & 7 \\
\hline Settin et al. & 2011 & Egypt & case-control & Africa & $72 / 70$ & 19 to $38 / 19$ to 38 & PCR & 6 \\
\hline Dissanayake et al. [32] & 2012 & Sri Lanka & case-control & Asia & $200 / 200$ & $32.1 \pm 5.6 / 32.4 \pm 4.6$ & RLFP-PCR & 8 \\
\hline Gazi et al. & 2012 & Turkey & case-control & Europe & $57 / 47$ & $30.12 \pm 7.32 / 27.80 \pm 6.36$ & PCR & 6 \\
\hline Karata et al. & 2012 & Turkey & case-control & Europe & $84 / 84$ & $31.6 \pm 3.7 / 32.2 \pm 3.9$ & $Q-P C R$ & 6 \\
\hline Mierla et al. [48] & 2012 & Romania & case-control & Europe & 283/100 & $33.76 / 32.8$ & RLFP-PCR & 7 \\
\hline Ozdemir et al. [49] & 2012 & Turkey & case-control & Europe & $543 / 106$ & $27.8 \pm 2.1 / 28.9 \pm 2.2$ & Q-PCR & 7 \\
\hline Torabi et al. [50] & 2012 & Iran & case-control & Asia & $100 / 100$ & $N R / N R$ & RLFP-PCR & 6 \\
\hline Kaur et al. & 2012 & India & case-control & Asia & $107 / 588$ & $24.89 / 25.32$ & RLFP-PCR & 7 \\
\hline Parveen et al. & 2012 & India & case-control & Asia & $1000 / 500$ & $28.4 \pm 5.9 / 31.9 \pm 7.3$ & ARMS-PCR & 8 \\
\hline Ardestani et al. & 2012 & Iran & case-control & Asia & $80 / 80$ & $28.8 / 23.6$ & RLFP-PCR & 6 \\
\hline Cardona et al. [51] & 2012 & Colombia & case-control & South America & $93 / 206$ & $34.1 \pm 0.9 / 41.6 \pm 0.7$ & RLFP-PCR & 7 \\
\hline Kazerooni et al. [52] & 2013 & Iran & case-control & Asia & $60 / 60$ & $24.8 \pm 3.9 / 24.6 \pm 4.7$ & PCR & 5 \\
\hline Baumann et al. & 2013 & Germany & cohort & Europe & $641 / 157$ & $32.95 \pm 4.94 / 33.16 \pm 6.24$ & RLFP-PCR & 8 \\
\hline Parand et al. [53] & 2013 & Iran & case-control & Asia & $90 / 44$ & $29.21 \pm 5.9 / 28.75 \pm 5.2$ & RLFP-PCR & 6 \\
\hline Zonouzi et al. [54] & 2013 & Iran & case-control & Asia & $89 / 50$ & $30.18 \pm 4.95 / 31.54 \pm 4.81$ & ARMS-PCR & 6 \\
\hline
\end{tabular}


Table 1 Characteristics of studies included in meta-analysis (Continued)

\begin{tabular}{|c|c|c|c|c|c|c|c|c|}
\hline Study author & Year & Country & $\begin{array}{l}\text { Study } \\
\text { design }\end{array}$ & Ethnicity & $\begin{array}{l}\text { Total cases/ } \\
\text { controls }\end{array}$ & $\begin{array}{l}\text { Age } \\
\text { case/control (Mean) }\end{array}$ & $\begin{array}{l}\text { Genotyping } \\
\text { method }\end{array}$ & $\begin{array}{l}\text { Quality } \\
\text { score }\end{array}$ \\
\hline Dutra et al. & 2013 & Brazil & case-control & South America & $145 / 135$ & $31.72 / 29.86$ & Q-PCR & 6 \\
\hline Isaoglu et al. & 2013 & Turkey & case-control & Europe & $60 / 40$ & $29.14 \pm 6.18 / 30.50 \pm 6.77$ & NR & 6 \\
\hline Pietropolli et al. [55] & 2014 & Italy & case-control & Europe & 186/129 & $35.2 \pm 5.1 / 40.4 \pm 5.3$ & Rapid-cycle PCR & 7 \\
\hline Lino et al. & 2014 & Brazil & case-control & South America & $83 / 98$ & $30.3 / 40.2$ & $Q-P C R$ & 6 \\
\hline Sharma et al. [56] & 2015 & India & case-control & Asia & $78 / 78$ & $28.6 \pm 3.32 / 30.5 \pm 2.57$ & RLFP-PCR & 6 \\
\hline Farahmand et al. & 2015 & Iran & case-control & Asia & $330 / 350$ & $30.37 / 29.88$ & $P C R$ & 8 \\
\hline Kashif et al. [57] & 2015 & Pakistan & case-control & Asia & $56 / 56$ & $28.55 \pm 4.69 / 28.61 \pm 4.38$ & $P C R$ & 6 \\
\hline Gonçalves et al. [58] & 2016 & Brazil & case-control & South America & $137 / 100$ & $32.1 / 25.8$ & RLFP-PCR & 7 \\
\hline Khaniani et al. [59] & 2016 & Iran & case-control & Asia & $210 / 160$ & less than 40 / NR & RLFP-PCR & 7 \\
\hline Eldeen et al. & 2017 & Arabia & case-control & Asia & $96 / 96$ & $37.7 \pm 4.6 / 36.5 \pm 5.8$ & $P C R$ & 6 \\
\hline Wolski et al. [60] & 2017 & Poland & case-control & Europe & $359 / 400$ & $30.99 \pm 4.50 / 30.05 \pm 3.81$ & RLFP-PCR & 8 \\
\hline Elgari et al. [61] & 2017 & Arabia & case-control & Asia & $60 / 80$ & $38 \pm 12 / 38 \pm 12$ & Multiplex PCR & 6 \\
\hline Mahmutbegović et al. [62] & 2017 & Bosnia & case-control & Europe & $51 / 154$ & $32.9 \pm 5.1 / 31.7 \pm 6.6$ & Q-PCR & 6 \\
\hline Wingeyer et al. & 2017 & Argentina & case-control & South America & $247 / 107$ & $32 / N R$ & Q-PCR & 7 \\
\hline Jusić et al. & 2018 & Bosnia & case-control & Europe & $60 / 80$ & $33.05 / 34.08$ & RLFP-PCR & 6 \\
\hline Taghi Kardi et al. & 2018 & Iran & case-control & Asia & $250 / 116$ & $29.7 \pm 3.4 / 30.4 \pm 3.2$ & Multiplex PCR & 7 \\
\hline Xu et al. & 2018 & China & case-control & Asia & $426 / 444$ & $29.26 \pm 4.294 / 34.50 \pm 4.895$ & Multiplex PCR & 8 \\
\hline Bigdeli et al. [63] & 2018 & Iran & case-control & Asia & $200 / 200$ & $23.0 \pm 3.8 / 25.1 \pm 4.4$ & RLFP-PCR & 8 \\
\hline Reddy et al. [64] & 2019 & India & case-control & Asia & $50 / 28$ & $26.8 / 27.6$ & RLFP-PCR & 5 \\
\hline Yengel et al. & 2019 & turkey & case-control & Europe & $145 / 105$ & $30.5 \pm 6.5 / 30.5 \pm 6.7$ & real-time PCR & 6 \\
\hline
\end{tabular}

total of 1266 studies were retrieved (PubMed: 254, Scopus: 512, and ISI Web of Science: 500). Subsequently, application of inclusion/exclusion criteria resulted in the exclusion of 1206 studies (324 duplicates studies, 714 and 168 studies excluded according to title \& abstract and full-text examination, respectively). Eventually, 62 qualified studies were included in the quantitative analysis, of which two studies were detected by cross-check of eligible studies and reviews [32, 33]. All eligible studies were published between 1999 to 2019 and had an overall good methodological quality with NOS scores ranging from 5 to 8 . The Restriction fragment length polymorphism (RFLP)-PCR was the most genotyping methods which used in the included studies. Except two studies which had cohort design, other $\mathbf{6 0}$ studies had case-control design. Tables $\mathbf{1}$ and $\mathbf{2}$ summarize the characteristics and allele/genotype frequency of the included studies.

\section{Meta-analysis of FVL $1691 \mathrm{G}>\mathrm{A}$ mutation and the risk of RPL}

Overall, 62 studies with 10,410 cases and 9406 controls included in quantitative analysis of the association between FVL 1691G > A mutation and the risk of RPL. Of those, 25 studies were in Asian countries [21, 22, 32, 35, $38,43,44,47,50,52-54,56,57,59,61,63-71], 26$ studies were conducted in European countries [17, 33, 36, 37, 39, 41, 42, 45, 48, 49, 55, 60, 62, 72-82], 6 studies in South American countries [34, 51, 58, 83-85], 4 studies in African countries [40, 46, 86, 87] and one study in Oceania. The analysis of overall population revealed a significant positive association between FVL 1691G > A mutation and the risk of RPL across all possible genotype models, including dominant model $(\mathrm{OR}=2.15,95 \%$ $\mathrm{CI}=1.84-2.50, P<0.001, \mathrm{FEM})$, over-dominant model $(\mathrm{OR}=1.88,95 \% \mathrm{CI}=1.61-2.19, P<0.001$, FEM $)$, allelic model $(\mathrm{OR}=2.05,95 \% \mathrm{CI}=1.79-2.35, P<0.001$, REM $)$, and heterozygote model $(\mathrm{OR}=1.97,95 \% \mathrm{CI}=1.68-2.30$, $P<0.001$, FEM) (Table 3 and Fig. 2).

\section{Meta-analysis of FVL $1691 \mathrm{G}>\mathrm{A}$ mutation and the risk of RPL in Iranian population}

Among the included studies, studies performed in Iran with 9 publications (1409 cases and 1160 controls) were in the first rank with respect to sample size and the number of studies, therefore we performed separate analysis. Our results found a significant association between FVL 1691G > A mutation and increased risk of RPL in this population under dominant model (OR $=3.04,95 \%$ $\mathrm{CI}=2.04-4.54, \quad P<0.001, \mathrm{FEM})$, over-dominant model $(\mathrm{OR}=2.65,95 \% \mathrm{CI}=1.74-4.05, \quad P<0.001, \quad \mathrm{FEM})$, and heterozygote model $(\mathrm{OR}=2.67,95 \% \mathrm{CI}=1.81-4.22, \mathrm{P}<$ 
Table 2 Distribution of genotype and allele among RPL patients and controls

\begin{tabular}{|c|c|c|c|c|c|c|c|c|c|c|c|c|}
\hline \multirow[t]{2}{*}{ Study author } & \multicolumn{5}{|c|}{ RPL cases } & \multicolumn{5}{|c|}{ Healthy control } & \multirow[t]{2}{*}{ P-HWE } & \multirow[t]{2}{*}{ MAF } \\
\hline & $\overline{G G}$ & GA & $\mathrm{AA}$ & G & A & GG & GA & $\mathrm{AA}$ & G & $A$ & & \\
\hline Souza et al. [34] & 52 & 4 & 0 & 108 & 4 & 378 & 6 & 0 & 762 & 6 & $0 / 87$ & $0 / 007$ \\
\hline Brenner et al. [35] & 52 & 19 & 5 & 123 & 29 & 95 & 11 & 0 & 201 & 11 & $0 / 57$ & $0 / 051$ \\
\hline Wramsby et al. [36] & 51 & 10 & 1 & 112 & 12 & 67 & 2 & 0 & 136 & 2 & $0 / 9$ & 0/014 \\
\hline Murphy et al. [52] & 39 & 2 & 0 & 80 & 2 & 527 & 13 & 0 & 1067 & 13 & $0 / 77$ & $0 / 012$ \\
\hline Pihusch et al. [33] & 94 & 8 & 0 & 196 & 8 & 117 & 11 & 0 & 245 & 11 & $0 / 61$ & $0 / 042$ \\
\hline Younis et al. & 63 & 12 & 3 & 138 & 18 & 131 & 8 & 0 & 270 & 8 & $0 / 72$ & $0 / 028$ \\
\hline Foka et al. [14] & 65 & 15 & 0 & 145 & 15 & 96 & 4 & 0 & 196 & 4 & $0 / 83$ & $0 / 02$ \\
\hline Rai et al. & 1037 & 72 & 2 & 2146 & 76 & 138 & 12 & 0 & 288 & 12 & $0 / 6$ & $0 / 04$ \\
\hline Carp et al. & 104 & 4 & 0 & 212 & 4 & 77 & 5 & 0 & 159 & 5 & $0 / 77$ & $0 / 03$ \\
\hline Finan et al. [38] & 65 & 38 & 7 & 168 & 52 & 56 & 11 & 0 & 123 & 11 & $0 / 46$ & 0/082 \\
\hline Hohlagschwandtner et al. & 130 & 15 & 0 & 275 & 15 & 97 & 4 & 0 & 198 & 4 & $0 / 83$ & $0 / 019$ \\
\hline Pauer et al. [39] & 28 & 2 & 0 & 58 & 2 & 113 & 9 & 0 & 235 & 9 & $0 / 67$ & $0 / 036$ \\
\hline Mtiraoui et al. & 116 & 24 & 6 & 256 & 36 & 93 & 6 & 0 & 192 & 6 & $0 / 75$ & $0 / 03$ \\
\hline Aksoy et al. & 31 & 9 & 1 & 71 & 11 & 45 & 5 & 0 & 95 & 5 & $0 / 7$ & $0 / 05$ \\
\hline Mahjoub et al. [40] & 152 & 40 & 8 & 344 & 56 & 189 & 11 & 0 & 389 & 11 & $0 / 68$ & $0 / 027$ \\
\hline Ulukus et al. & 7 & 3 & 0 & 17 & 3 & 49 & 3 & 1 & 101 & 5 & $\leq 0.001$ & $0 / 047$ \\
\hline Sotiriadis et al. & 94 & 5 & 0 & 193 & 5 & 99 & 3 & 0 & 201 & 3 & $0 / 88$ & 0/014 \\
\hline Mohammad et al. [21] & 25 & 10 & 0 & 60 & 10 & 41 & 4 & 0 & 86 & 4 & $0 / 75$ & $0 / 044$ \\
\hline Altintas et al. [41] & 105 & 9 & 0 & 219 & 9 & 172 & 13 & 0 & 357 & 13 & $0 / 62$ & $0 / 035$ \\
\hline Toth et al. [42] & 138 & 13 & 0 & 289 & 13 & 145 & 12 & 0 & 302 & 12 & $0 / 61$ & 0/038 \\
\hline Pasquier et al. & 296 & 15 & 0 & 607 & 15 & 574 & 25 & 0 & 1173 & 25 & $0 / 6$ & $0 / 02$ \\
\hline Biswas et al. [43] & 83 & 2 & 0 & 168 & 2 & 31 & 0 & 0 & 62 & 0 & $\leq 0.001$ & 0 \\
\hline Lvanov et al. & 133 & 19 & 1 & 285 & 21 & 93 & 7 & 0 & 193 & 7 & $0 / 71$ & $0 / 035$ \\
\hline Mukhopadhyay et al. [44] & 80 & 4 & 0 & 164 & 4 & 80 & 0 & 0 & 160 & 0 & $\leq 0.001$ & 0 \\
\hline Ciacci et al. [45] & 38 & 1 & 0 & 77 & 1 & 70 & 2 & 0 & 142 & 2 & $0 / 9$ & 0/013 \\
\hline Mohamed et al. [46] & 6 & 12 & 2 & 24 & 16 & 19 & 1 & 0 & 39 & 1 & $0 / 9$ & $0 / 025$ \\
\hline Hussein et al. [47] & 104 & 36 & 5 & 244 & 46 & 181 & 24 & 0 & 386 & 24 & $0 / 37$ & 0/058 \\
\hline Serrano et al. [17] & 95 & 5 & 0 & 195 & 5 & 95 & 5 & 0 & 195 & 5 & $0 / 79$ & $0 / 025$ \\
\hline Settin et al. & 54 & 17 & 1 & 125 & 19 & 69 & 1 & 0 & 139 & 1 & $0 / 95$ & $0 / 007$ \\
\hline Dissanayake et al. [32] & 196 & 4 & 0 & 396 & 4 & 195 & 5 & 0 & 395 & 5 & $0 / 85$ & $0 / 012$ \\
\hline Gazi et al. & 50 & 6 & 1 & 106 & 8 & 43 & 4 & 0 & 90 & 4 & $0 / 76$ & $0 / 042$ \\
\hline Karata et al. & 66 & 16 & 2 & 148 & 20 & 66 & 18 & 0 & 150 & 18 & $0 / 27$ & $0 / 107$ \\
\hline Mierla et al. [48] & 260 & 21 & 2 & 541 & 25 & 95 & 5 & 0 & 195 & 5 & $0 / 79$ & $0 / 025$ \\
\hline Ozdemir et al. [49] & 433 & 109 & 1 & 975 & 111 & 104 & 2 & 0 & 210 & 2 & $0 / 92$ & $0 / 009$ \\
\hline Torabi et al. [50] & 87 & 12 & 1 & 186 & 14 & 96 & 4 & 0 & 196 & 4 & $0 / 83$ & $0 / 02$ \\
\hline Kaur et al. & 102 & 4 & 1 & 208 & 6 & 573 & 15 & 0 & 1161 & 15 & $0 / 75$ & $0 / 012$ \\
\hline Parveen et al. & 950 & 50 & 0 & 1950 & 50 & 488 & 12 & 0 & 988 & 12 & $0 / 78$ & 0/012 \\
\hline Ardestani et al. & 78 & 2 & 0 & 158 & 2 & 79 & 1 & 0 & 159 & 1 & $0 / 95$ & $0 / 006$ \\
\hline Cardona et al. [51] & 92 & 1 & 0 & 185 & 1 & 205 & 1 & 0 & 411 & 1 & $0 / 97$ & $0 / 002$ \\
\hline Kazerooni et al. [52] & 43 & 12 & 5 & 98 & 22 & 54 & 4 & 2 & 112 & 8 & 0.48 & 0.734 \\
\hline Baumann et al. & 592 & 49 & 0 & 1233 & 49 & 145 & 12 & 0 & 302 & 12 & $0 / 61$ & 0/038 \\
\hline Parand et al. [53] & 72 & 15 & 3 & 159 & 21 & 38 & 6 & 0 & 82 & 6 & $0 / 62$ & 0/068 \\
\hline Zonouzi et al. [54] & 87 & 2 & 0 & 176 & 2 & 50 & 0 & 0 & 100 & 0 & $\leq 0.001$ & 0 \\
\hline
\end{tabular}


Table 2 Distribution of genotype and allele among RPL patients and controls (Continued)

\begin{tabular}{|c|c|c|c|c|c|c|c|c|c|c|c|c|}
\hline \multirow[t]{2}{*}{ Study author } & \multicolumn{5}{|c|}{ RPL cases } & \multicolumn{5}{|c|}{ Healthy control } & \multirow[t]{2}{*}{ P-HWE } & \multirow[t]{2}{*}{ MAF } \\
\hline & GG & GA & AA & G & A & GG & GA & $\mathrm{AA}$ & G & A & & \\
\hline Dutra et al. & 142 & 3 & 0 & 287 & 3 & 131 & 4 & 0 & 266 & 4 & $0 / 86$ & $0 / 014$ \\
\hline Isaoglu et al. & 47 & 13 & 0 & 107 & 13 & 39 & 1 & 0 & 79 & 1 & 0/93 & 0/012 \\
\hline Pietropolli et al. [55] & 168 & 18 & 0 & 354 & 18 & 125 & 4 & 0 & 254 & 4 & 0/85 & 0/015 \\
\hline Lino et al. & 79 & 4 & 0 & 162 & 4 & 96 & 2 & 0 & 194 & 2 & 0/91 & 0/01 \\
\hline Sharma et al. [56] & 36 & 40 & 2 & 112 & 44 & 77 & 1 & 0 & 155 & 1 & 0/95 & 0/006 \\
\hline Farahmand et al. & 302 & 28 & 0 & 632 & 28 & 340 & 10 & 0 & 690 & 10 & 0/78 & 0/014 \\
\hline Kashif et al. [57] & 53 & 3 & 0 & 109 & 3 & 56 & 0 & 0 & 112 & 0 & $\leq 0.001$ & 0 \\
\hline Gonçalves et al. [58] & 133 & 4 & 0 & 270 & 4 & 98 & 2 & 0 & 198 & 2 & $0 / 91$ & 0/01 \\
\hline Khaniani et al. [59] & 202 & 8 & 0 & 412 & 8 & 158 & 2 & 0 & 318 & 2 & 0/93 & 0/006 \\
\hline Eldeen et al. & 0 & 72 & 24 & 72 & 120 & 0 & 94 & 2 & 94 & 98 & $\leq 0.001$ & $0 / 51$ \\
\hline Wolski et al. [60] & 333 & 26 & 0 & 692 & 26 & 378 & 21 & 1 & 777 & 23 & $0 / 23$ & $0 / 028$ \\
\hline Elgari et al. [61] & 56 & 4 & 0 & 116 & 4 & 74 & 6 & 0 & 154 & 6 & $0 / 72$ & 0/037 \\
\hline Mahmutbegović et al. [62] & 44 & 7 & 0 & 95 & 7 & 142 & 12 & 0 & 296 & 12 & $0 / 61$ & 0/038 \\
\hline Wingeyer et al. & 239 & 8 & 0 & 486 & 8 & 105 & 2 & 0 & 212 & 2 & 0/92 & 0/009 \\
\hline Jusić et al. & 51 & 9 & 0 & 111 & 9 & 77 & 3 & 0 & 157 & 3 & $0 / 86$ & 0/018 \\
\hline Taghi Kardi et al. & 236 & 12 & 2 & 484 & 16 & 109 & 5 & 2 & 223 & 9 & $\leq 0.001$ & 0/038 \\
\hline Xu et al. & 426 & 0 & 0 & 852 & 0 & 443 & 1 & 0 & 887 & 1 & 0/98 & 0/001 \\
\hline Bigdeli et al. [63] & 150 & 30 & 20 & 330 & 70 & 192 & 8 & 0 & 392 & 8 & 0/77 & 0/02 \\
\hline Yengel et al. & 130 & 1 & 14 & 261 & 29 & 102 & 0 & 3 & 204 & 6 & 0/65 & $0 / 394$ \\
\hline
\end{tabular}

$P$-HWE $p$-value for Hardy-Weinberg equilibrium; MAF Minor allele frequency of control group

0.001, FEM) but not allelic model $(\mathrm{OR}=2.09,95 \% \mathrm{CI}=$ 0.88-4.94, $P=0.09$, REM) (Table 3).

\section{Subgroup analysis by continent}

The included studies were performed in Asia (25 studies), Europe (26 studies), South America (6 studies), Africa (4 studies) and Oceania (1 article). Since there was only one study for Oceania, we exclude it from the subgroup analysis. The final results revealed strong significant association between FVL 1691G > A mutation and the risk of RPL in Asian, European, and Africa population, but not in South Americans (Fig. 3). The results of pooled ORs, heterogeneity tests, and publication bias tests in different analysis models are shown in the Table 3.

\section{Subgroup analysis by study design}

The stratification of studies based on study design caused to the inclusion of two studies with 1752 cases and 307 controls in cohort group, and 60 studies with 8658 cases and 9099 controls in case-control group. The findings demonstrated a statistical significant association between FVL 1691G > A mutation and the risk of RPL in case-control studies across dominant model $(\mathrm{OR}=2.33,95 \% \mathrm{CI}=1.99-2.74, P<$ 0.001 , FEM), over-dominant model $(\mathrm{OR}=2.05,95 \%$ $\mathrm{CI}=1.74-2.41, \quad P<0.001, \mathrm{FEM})$, allelic model $(\mathrm{OR}=$
2.18, 95\% CI $=1.8-2.52, P<0.001$, FEM), and heterozygote model $(\mathrm{OR}=2.16,95 \% \mathrm{CI}=1.83-2.55, \quad P<$ 0.001, FEM). However, no significant association was observed in cohort studies (Table 3 ).

\section{Heterogeneity and publication bias}

To check existence of publication bias, Egger's linear regression and Begg's funnel plot test were used. The shape of the funnel plots did not disclose obvious asymmetry under all the genotype model of the FVL 1691G > A mutation (Fig. 4). Additionally, some degree of heterogeneity was detected in overall population. Therefore, we stratified study by continent and study design to find its potential source.

\section{Meta-regression analyses}

Meta-regression analyses were performed to explore potential sources of heterogeneity among included studies (Table 4). The findings indicated that none of the expected heterogeneity parameter were the source of heterogeneity (Fig. 5).

\section{Sensitivity analysis}

The impact of individual study on pooled OR was evaluated by sequential omission of each studies. The analysis results showed that no individual study significantly affected the pooled ORs under any genotype models of the FVL 1691G > A mutation (Fig. 6). 
Table 3 Main results of pooled ORs in meta-analysis of FVL 1691G > A mutation

\begin{tabular}{|c|c|c|c|c|c|c|c|c|c|c|}
\hline \multirow[t]{2}{*}{ Subgroup } & \multirow[b]{2}{*}{ Genetic model } & \multirow{2}{*}{$\begin{array}{l}\text { Sample size } \\
\text { Case/Control }\end{array}$} & \multicolumn{2}{|c|}{ Test of association } & \multicolumn{2}{|c|}{$\begin{array}{l}\text { Test of } \\
\text { heterogeneity }\end{array}$} & \multicolumn{2}{|c|}{$\begin{array}{l}\text { Test of publication } \\
\text { bias (Begg's test) }\end{array}$} & \multicolumn{2}{|c|}{$\begin{array}{l}\text { Test of publication } \\
\text { bias (Egger's test) }\end{array}$} \\
\hline & & & OR & $95 \% \mathrm{Cl}$ ( $P$-value) & OR & $P$ & Z & $P$ & $\mathbf{T}$ & $P$ \\
\hline \multirow[t]{4}{*}{ Overall } & Dominant & 10,410 / 9406 & 2.15 & $1.84-2.50(<0.001)$ & 38.3 & 0.002 & 1.49 & 0.13 & 1.64 & 0.11 \\
\hline & Over-Dominant & $10,410 / 9406$ & 1.88 & $1.61-2.19(<0.001)$ & 35.8 & 0.005 & 1.33 & 0.17 & 1.45 & 0.14 \\
\hline & Allelic model & $10,410 / 9406$ & 2.05 & $1.79-2.35(<0.001)$ & 48.6 & $\leq 0.001$ & 1.45 & 0.16 & 1.59 & 0.13 \\
\hline & GA vs. GG & $10,410 / 9406$ & 1.97 & $1.68-2.30(<0.001)$ & 28.3 & 0.03 & 1.51 & 0.11 & 2.01 & 0.04 \\
\hline \multirow[t]{4}{*}{ Iranian population } & Dominant & $1409 / 1160$ & 3.04 & $2.04-4.54(<0.001)$ & 37.3 & 0.13 & -0.45 & 0.65 & -0.53 & 0.61 \\
\hline & Over-Dominant & $1409 / 1160$ & 2.65 & $1.74-4.05(<0.001)$ & 0 & 0.66 & -1.05 & 0.29 & -0.64 & 0.55 \\
\hline & Allelic model & $1409 / 1160$ & 2.09 & $0.88-4.94(<0.09)$ & 76.8 & 0.008 & -0.45 & 0.65 & -0.33 & 0.75 \\
\hline & GA vs. GG & $1409 / 1160$ & 2.67 & $1.81-4.22(<0.001)$ & 0 & 0.59 & -1.05 & 0.29 & -0.67 & 0.53 \\
\hline \multicolumn{11}{|c|}{ Subgroup (continent) } \\
\hline \multirow[t]{4}{*}{ Asia } & Dominant & 4153 / 3957 & 2.80 & $2.20-3.56(<0.001)$ & 35.4 & 0.06 & -0.80 & 0.42 & -0.44 & 0.64 \\
\hline & Over-Dominant & 4153 / 3957 & 2.22 & $1.73-2.85(<0.001)$ & 47.2 & 0.01 & -1.43 & 0.15 & -0.98 & 0.34 \\
\hline & Allelic model & 4153 / 3957 & 2.35 & $1.92-2.87(<0.001)$ & 62.6 & 0.003 & -0.45 & 0.64 & 0.39 & 0.7 \\
\hline & GA vs. GG & 4153 / 3957 & 2.51 & $1.95-3.21(<0.001)$ & 11.9 & 0.31 & -1.10 & 0.27 & -0.35 & 0.73 \\
\hline \multirow[t]{4}{*}{ Europe } & Dominant & 4913 / 3929 & 1.49 & $1.20-1.84(0.001)$ & 14.7 & 0.25 & 3.06 & 0.002 & 3.79 & 0.001 \\
\hline & Over-Dominant & 4913 / 3929 & 1.43 & $1.15-1.79(0.002)$ & 16 & 0.23 & 2.99 & 0.003 & 3.65 & 0.001 \\
\hline & Allelic model & 4913 / 3929 & 1.48 & $1.19-1.81(0.001)$ & 9.9 & 0.32 & 1.37 & 0.16 & 1.58 & 0.13 \\
\hline & GA vs. GG & 4913 / 3929 & 1.44 & $1.15-1.80(0.001)$ & 16 & 0.23 & 2.96 & 0.003 & 3.65 & 0.001 \\
\hline \multirow[t]{4}{*}{ South America } & Dominant & $761 / 1030$ & 2.04 & $0.88-4.74(0.09)$ & 0 & 0.76 & 0.19 & 0.85 & -0.53 & 0.62 \\
\hline & Over-Dominant & $761 / 1030$ & 2.04 & $0.88-4.74(0.09)$ & 0 & 0.76 & 0.19 & 0.85 & -0.53 & 0.62 \\
\hline & Allelic model & $761 / 1030$ & 2 & $0.87-4.60(0.1)$ & 0 & 0.76 & -0.19 & 0.85 & -0.47 & 0.66 \\
\hline & GA vs. GG & $761 / 1030$ & 2.04 & $0.88-4.74(0.09)$ & 0 & 0.76 & 0.19 & 0.85 & -0.53 & 0.62 \\
\hline \multirow[t]{4}{*}{ Africa } & Dominant & 438 / 389 & 5.65 & $3.15-10.14(<0.001)$ & 3.9 & 0.37 & 1.36 & 0.17 & 2.55 & 0.12 \\
\hline & Over-Dominant & 438 / 389 & 4.44 & $2.45-8.03(<0.001)$ & 3.2 & 0.37 & 1.36 & 0.17 & 2.41 & 0.13 \\
\hline & Allelic model & 438 / 389 & 5.93 & $3.38-10.40(<0.001)$ & 0 & 0.55 & 1.36 & 0.17 & 2.59 & 0.12 \\
\hline & GA vs. GG & 438 / 389 & 4.70 & $2.59-8.53(<0.001)$ & 12.3 & 0.33 & 1.36 & 0.17 & 2.47 & 0.13 \\
\hline \multicolumn{11}{|c|}{ Subgroup (Study design) } \\
\hline \multirow[t]{4}{*}{ Case-Control } & Dominant & 8658 / 9099 & 2.33 & $1.99-2.74(<0.001)$ & 31.5 & 0.01 & 0.28 & 0.78 & 0.79 & 0.43 \\
\hline & Over-Dominant & 8658 / 9099 & 2.05 & $1.74-2.41(<0.001)$ & 29.3 & 0.02 & 1.43 & 0.15 & 1.28 & 0.21 \\
\hline & Allelic model & 8658 / 9099 & 2.18 & $1.8-2.52(<0.001)$ & 44.9 & 0.003 & 0.71 & 0.47 & 0.87 & 0.39 \\
\hline & GA vs. GG & $8658 / 9099$ & 2.16 & $1.83-2.55(<0.001)$ & 18.2 & 0.13 & 1.70 & 0.09 & 1.78 & 0.08 \\
\hline \multirow[t]{4}{*}{ Cohort } & Dominant & 1752 / 307 & 0.90 & $0.55-1.49(0.68)$ & 0 & 0.69 & -1.23 & 0.47 & -1.88 & 0.11 \\
\hline & Over-Dominant & $1752 / 307$ & 0.88 & $0.54-1.46(0.63)$ & 0 & 0.64 & -1.23 & 0.47 & -0.95 & 0.38 \\
\hline & Allelic model & $1752 / 307$ & 0.91 & $0.56-1.49(0.71)$ & 0 & 0.72 & -1.23 & 0.47 & -1.27 & 0.25 \\
\hline & GA vs. GG & 1752 / 307 & 0.88 & $0.54-1.46(0.63)$ & 0 & 0.64 & -1.23 & 0.47 & -1.68 & 0.14 \\
\hline
\end{tabular}

\section{Discussion}

RPL has been one of the most prevalent obstetric complications, that affect more than $30 \%$ of gestations. A remarkable amount of pregnancy losses has been attributed to genetic variations, of which over $50 \%$ have been related to chromosomal abnormalities. Several investigations have reported the association of FVL 1691G > A mutation with RPL; that notwithstanding, there have been conflicting results among various ethnicities. The inconsistent results have been attributed to variety in the race of included subjects, different diagnostic criteria of patients, little statistical power, small sample sizes, and the linkage disequilibrium (LD) between various genes and variations [88]. However, metaanalysis strategy provides a pertinent tool to settle the problem of confliction by resolving the limitations of single replication studies, such as limited statistical power and little sample size. Thus, here we conducted 


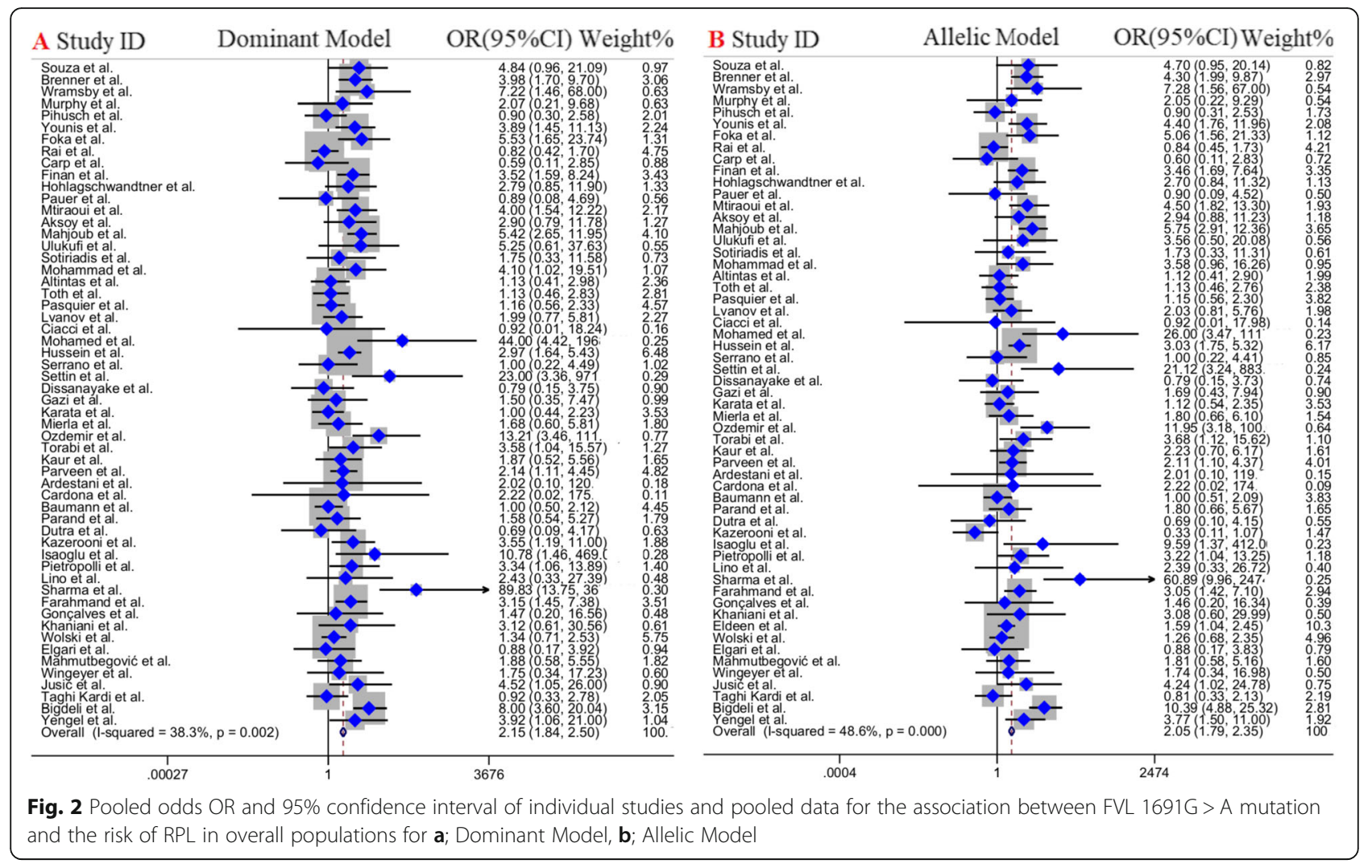

the first meta-analysis to find a valid estimation of the association between FVL 1691G > A mutation and risk of RPL.

The FVL 1691G > A mutation is a G-to-A point mutation at nucleotide 1691 in the factor $\mathrm{V}$ gene, that results in the single amino-acid replacement Arg506Gln, leading to resistance to be cleaved and, therefore, inactivation by APC and promoted susceptibility to clotting [89, 90]. This mutation enhances the risk of venous thrombosis up to 50-100 times in homozygote carriers [22].

In this meta-analysis, 62 studies, containing 10,410 cases and 9406 controls, were included in quantitative analysis. The analysis of overall population indicated that all genetic comparisons of the FVL 1691G > A mutation, including dominant model $(\mathrm{OR}=2.15)$, over-dominant model $(\mathrm{OR}=1.88)$, allelic model $(\mathrm{OR}=2.05)$, and heterozygote model $(\mathrm{OR}=1.97)$ significantly increased the risk of RPL susceptibility. In 2015, Sergi et al. [91] by including nine studies, containing a total of 2147 women for the FVL mutation, 1305 women with early RPL, and 842 women with no gestational complications, indicated higher carrier frequency of FVL mutation in women with early RPL $(\mathrm{OR}=1.68)$. Moreover, Marcelo and colleagues [92] in 2019 revealed that there was no association between recurrent miscarriage and inherited thrombophilias in patients with polycystic ovarian syndrome, with respect to FVL $(\mathrm{OR}=0.74 ; 95 \% \mathrm{CI}=0.38-$
1.45; $P=0.38$ ), among others. On the other hand, a comprehensive systematic review and meta-analysis in 2016 [93], by exerting 369 articles evaluating 124 polymorphisms of 73 genes, to explore the potential genetic biomarkers for recurrent miscarriage identified increased risk of the disease in the recessive and over-dominant models, but a decreased risk in the dominant and allelic models for FVL 1691G > A mutation, both in overall analysis and subgroup analysis in Caucasians. Our analysis is unique of its type, as it included only patients having RPL diagnosis. Moreover, our subgroup analysis based on the continent of the study population divulged a strong association between FVL 1691G > A mutation and the risk of RPL in Asian, European, and Africa populations, but not in South Americans. It should be noted that among the 62 case-control studies included, 25 studies were in Asia, 26 studies in Europe, 6 studies in South America, 4 studies in Africa, and 1 study in Oceania. Although the subgroup analysis of 6 studies in South America indicated an OR $<1$ (which was not significant across all genetic models), all other populations (which made large portion of the studies included) had $\mathrm{OR}>1$, imply that the South America data had little effect on the pooled effect estimation. The other parameter for subgroup analysis was study design. In this regard, a significant positive association between FVL 1691G > A mutation and the risk of 


\begin{tabular}{|c|c|c|c|}
\hline study ID & Over-Dominant model & OR $(95 \% \mathrm{CI})$ & Weight $\%$ \\
\hline $\begin{array}{l}\text { America } \\
\text { Souza et al. } \\
\text { Cardona et al. } \\
\text { Dutra et al. } \\
\text { Lino et al. } \\
\text { Gonçalves et al. } \\
\text { Wingeyer et al. } \\
\text { Subtotal (I-squared = }\end{array}$ & - & $\begin{array}{l}4.84(0.96,21.09) \\
2.22(0.02,175.06) \\
0.69(0.09,4.17) \\
2.43(0.33,27.39) \\
1.47(0.20,16.56) \\
1.75(0.34,17.23) \\
2.04(0.88,4.74)\end{array}$ & $\begin{array}{l}1.02 \\
0.12 \\
0.66 \\
0.50 \\
0.50 \\
0.63 \\
3.44\end{array}$ \\
\hline $\begin{array}{l}\text { Asia } \\
\text { Brenner et al. } \\
\text { Younis et al. } \\
\text { Carp et al. } \\
\text { Finan et al. } \\
\text { Mohammad et al. } \\
\text { Hussein et al. } \\
\text { Dissanaayake et al. } \\
\text { Torabi et al. } \\
\text { Kaur et al. } \\
\text { Parveen et al. } \\
\text { Ardestani et al. } \\
\text { Kazerooni et al. } \\
\text { Parand et al. } \\
\text { Sharma et al. } \\
\text { Farahmand et al. } \\
\text { Khaniani et al. } \\
\text { Eldeen et al. } \\
\text { Elgari et al. } \\
\text { Taghi Kardi et al. } \\
\text { Bigdeli et al. } \\
\text { Sübtotal (l-squared = }\end{array}$ & $-0.016)$ & 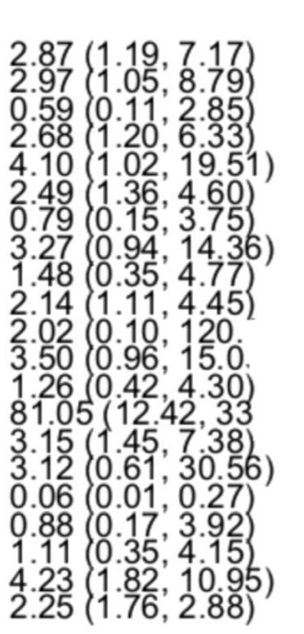 & $\begin{array}{l}3.03 \\
2.17 \\
0.92 \\
3.53 \\
1.12 \\
6.58 \\
0.94 \\
1.32 \\
1.43 \\
5.07 \\
0.19 \\
1.29 \\
1.81 \\
0.31 \\
3.69 \\
0.64 \\
0.73 \\
0.99 \\
1.60 \\
3.04 \\
40.42\end{array}$ \\
\hline $\begin{array}{l}\text { Europe } \\
\text { Wrramsby et al. } \\
\text { Murphy et al. } \\
\text { Pihusch et al. } \\
\text { Foka et al. } \\
\text { Rai et al. } \\
\text { Pauer et al. } \\
\text { Aksoy et al. } \\
\text { Uklukufi et al. } \\
\text { Sotiriadis et al. } \\
\text { Altintas et al. } \\
\text { Toth et al. } \\
\text { Pasquier et al. } \\
\text { Lvanov et al. } \\
\text { Ciacci et al. } \\
\text { Serrano et al. } \\
\text { Gazi et al. } \\
\text { Karata et al. } \\
\text { Mierla et al. } \\
\text { Ozdemir et al. } \\
\text { Baumann et al. } \\
\text { Isaoglu et al. } \\
\text { Pietropolli et al. } \\
\text { Wolski et al. } \\
\text { Mahmutbegović et al. } \\
\text { Jusić et al. } \\
\text { Subtotal (l-squared = }\end{array}$ & $0=0.236)$ & $\begin{array}{l}6.44(1.27,62.17) \\
2.07(0.21,9.68) \\
0.90(0.30,2.58) \\
5.53(1.65,23.74) \\
0.79(0.41,1.65) \\
0.89(0.08,4.69) \\
2.53(0.67,10.45) \\
7.14(0.76,61.75) \\
1.75(0.33,11.58) \\
1.13(0.41,2.98) \\
1.13(0.46,2.83) \\
1.16(0.56,2.33) \\
1.88(0.72,5.50) \\
0.92(0.01,18.24) \\
1.00(0.22,4.49) \\
1.26(0.27,6.48) \\
0.86(0.37,1.96) \\
1.52(0.53,5.31 \\
13.05(3.42,110 \\
1.00(0.50,2.12) \\
10.78(1.46,469 \\
3.34(1.06,13.89) \\
1.40(0.74,2.68) \\
1.88(0.58,5.55) \\
4.52(1.05,26.95) \\
1.43(1.15,1.79)\end{array}$ & $\begin{array}{l}0.65 \\
0.67 \\
2.11 \\
1.37 \\
5.04 \\
0.59 \\
1.30 \\
0.51 \\
0.77 \\
2.48 \\
2.96 \\
4.81 \\
2.36 \\
0.17 \\
1.07 \\
0.97 \\
3.52 \\
1.84 \\
0.81 \\
4.68 \\
0.29 \\
1.48 \\
5.90 \\
1.92 \\
0.93 \\
49.21\end{array}$ \\
\hline $\begin{array}{l}\text { Africa } \\
\text { Mtiraoui et al. } \\
\text { Mahjoub et al. } \\
\text { Mohamed et al. } \\
\text { Settin et al. } \\
\text { Subtotal (I-squared = }\end{array}$ & $=0.377)$ & $\begin{array}{l}3.04(1.14,9.45) \\
4.29(2.07,9.56) \\
28.50(2.98,128 \\
21.00(3.10,903 \\
4.44(2.45,8.03)\end{array}$ & $\begin{array}{l}2.19 \\
4.18 \\
0.27 \\
0.30 \\
6.93\end{array}$ \\
\hline Overall $(\mathrm{I}$-squared $=3$ & $=0.005)$ & $1.88(1.61,2.20)$ & 100.00 \\
\hline \multicolumn{2}{|l|}{$\begin{array}{c}1 \\
.0003\end{array}$} & \multicolumn{2}{|c|}{$\begin{array}{l}1 \\
3320\end{array}$} \\
\hline
\end{tabular}



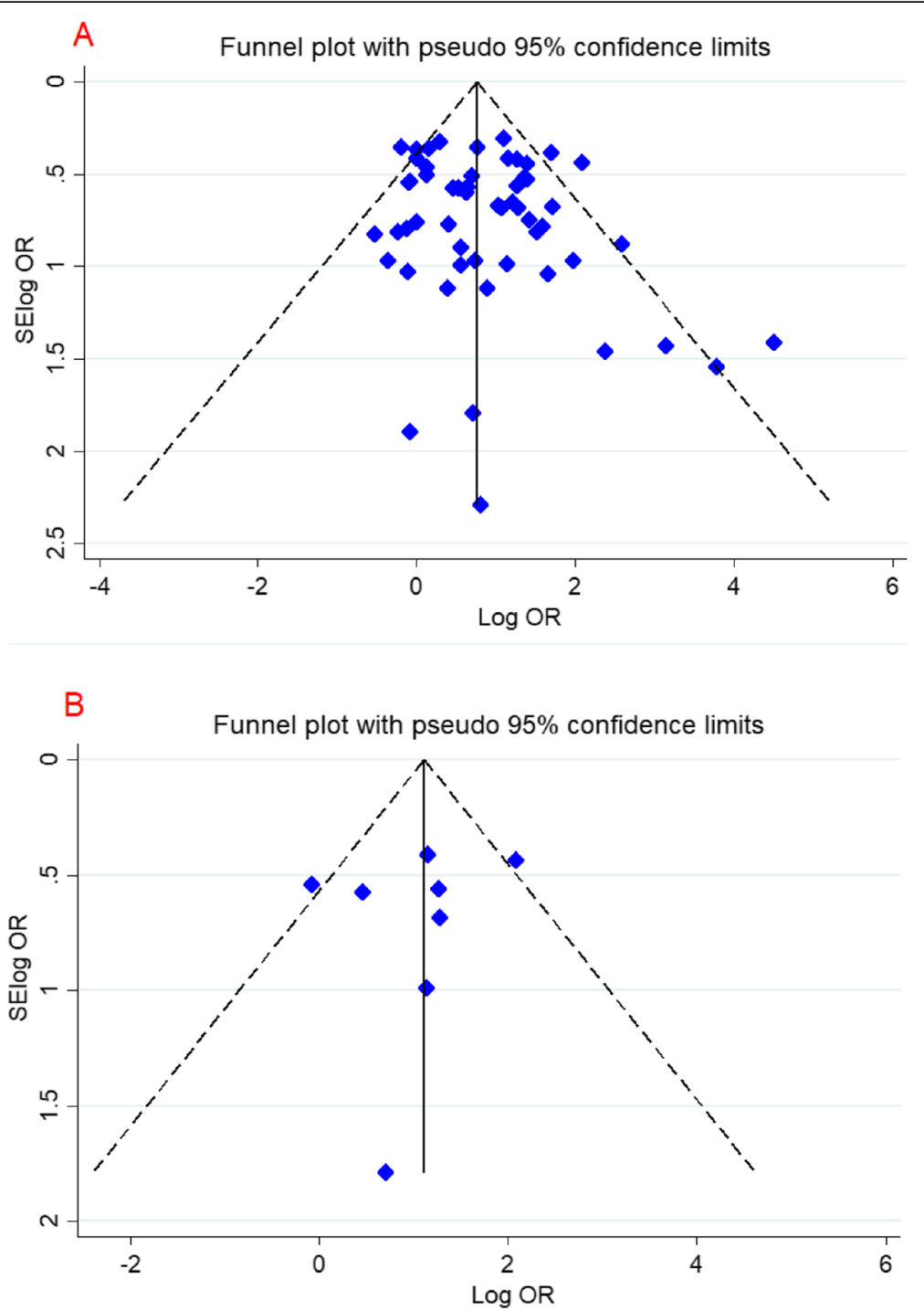

Fig. 4 Begg's funnel plot for publication bias test for the association between FVL 1691G > A mutation and the risk of RPL in the dominant model; a:overall population, b: Iranian studies. Each point represents a separate study for the indicated association

RPL was observed in case-control studies, while cohort studies revealed no such association. The result of this subgroup should interpret with caution because of imbalance between included studies in each group (60 vs. 2).

On the other side, the analysis was also performed in the Iranian population, containing 9 publications with 1409 cases and 1160 controls. The previous metaanalysis in Iranian population by Kamali et al. [94] in 2018 , by employing 7 studies, indicated significant increased risk of RPL only in the allelic $(\mathrm{OR}=2.252)$ and dominant models $(\mathrm{OR}=2.217)$. However, our analysis indicated that the measured genetic models, including dominant model $(\mathrm{OR}=2.97)$, over-dominant model $(\mathrm{OR}=2.58)$, and heterozygote model $(\mathrm{OR}=2.67,95 \%)$ increased the risk of RPL. The difference between our analysis and the previous one was that we included two more study with higher sample size.

There was a degree of heterogeneity during the overall analysis. From statistical perspective, this heterogeneity describes the variability between included studies and may originate from clinical or methodological heterogeneity, from other unreported, unknown study characteristics, or may be due to chance. Therefore, for finding any sources of heterogeneity and attenuating their effects, we conducted subgroup analysis and weighted meta-regression. Collectively, the results of metaregression showed that none of the parameters, including publication year, the continent of the study population, and genotyping methods were the expected source of heterogeneity. However, subgroup analysis reduced heterogeneity in all groups and explained part of the 
Table 4 Meta-regression analyses of potential source of heterogeneity

\begin{tabular}{|c|c|c|c|c|c|c|c|}
\hline \multirow[t]{2}{*}{ Heterogeneity Factor } & & \multirow[t]{2}{*}{ Coefficient } & \multirow[t]{2}{*}{ SE } & \multirow[t]{2}{*}{ T-test } & \multirow[t]{2}{*}{ P-value } & \multicolumn{2}{|l|}{$95 \% \mathrm{Cl}$} \\
\hline & & & & & & $U L$ & $\mathrm{LL}$ \\
\hline \multirow[t]{4}{*}{ Publication Year } & Dominant & 0.296 & 0.31 & 0.85 & 0.39 & -0.365 & 0.905 \\
\hline & Over-Dominant & 0.211 & 0.26 & 0.79 & 0.43 & -0.325 & 0.747 \\
\hline & Allelic model & 0.159 & 0.20 & 0.77 & 0.44 & -0.257 & 0.576 \\
\hline & GA vs. GG & 0.253 & 0.29 & 0.86 & 0.39 & -0.341 & 0.848 \\
\hline \multirow[t]{4}{*}{ Continent } & Dominant & 0.879 & 1.92 & 0.46 & 0.65 & -2.99 & 4.74 \\
\hline & Over-Dominant & 0.498 & 1.63 & 0.30 & 0.76 & -2.79 & 3.78 \\
\hline & Allelic model & 0.650 & 1.27 & 0.51 & 0.61 & -1.90 & 3.20 \\
\hline & GA vs. GG & 0.72 & 1.80 & 0.40 & 0.69 & -2.90 & 4.35 \\
\hline \multirow[t]{4}{*}{ Genotyping Methods } & Dominant & -0.04 & 1.35 & -0.04 & 0.97 & -2.76 & 2.66 \\
\hline & Over-Dominant & 0.028 & 1.15 & 0.02 & 0.98 & -2.29 & 2.35 \\
\hline & Allelic model & -0.115 & 0.89 & -0.13 & 0.89 & -1.92 & 1.68 \\
\hline & GA vs. GG & 0.016 & 1.26 & 0.01 & 0.98 & -2.52 & 2.55 \\
\hline
\end{tabular}

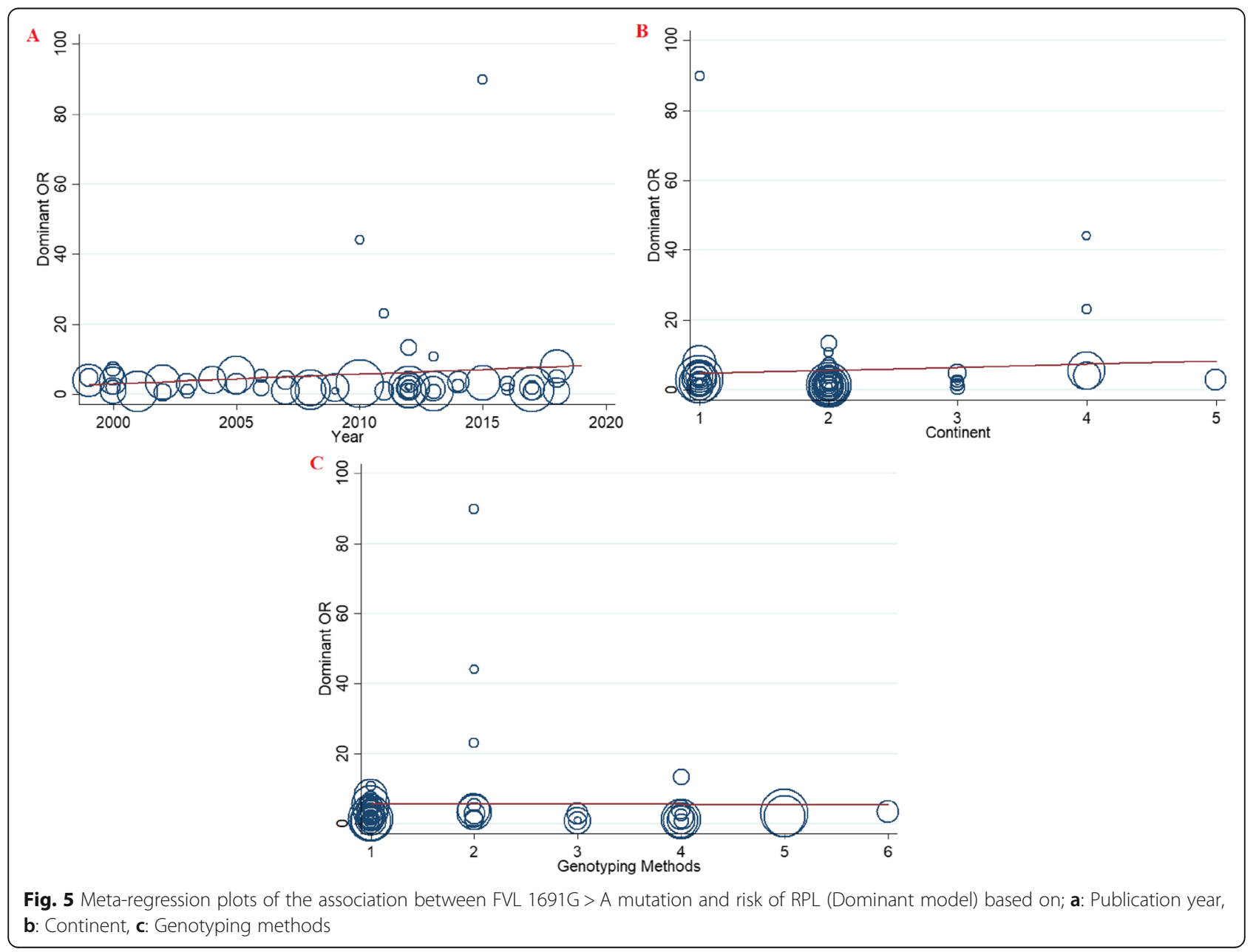


A Meta-analysis estimates, given named study is omitted

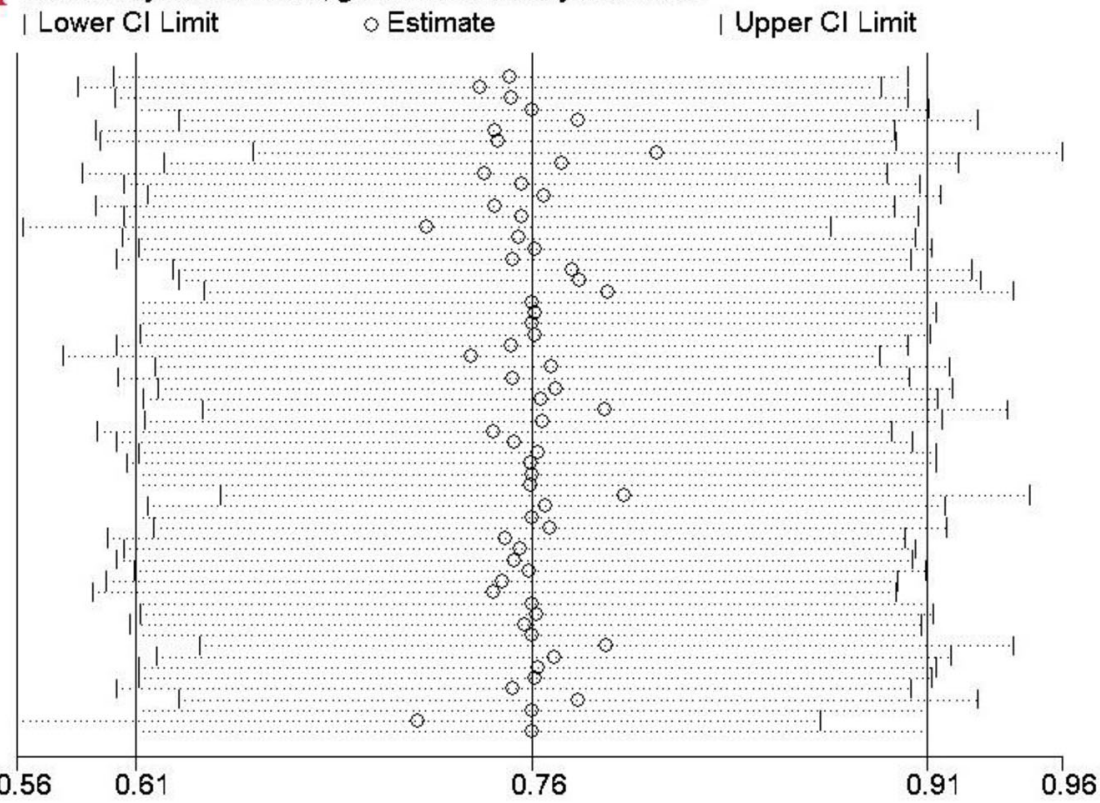

B

Meta-analysis estimates, given named study is omitted

I Lower Cl Limit ostimate

I Upper Cl Limit

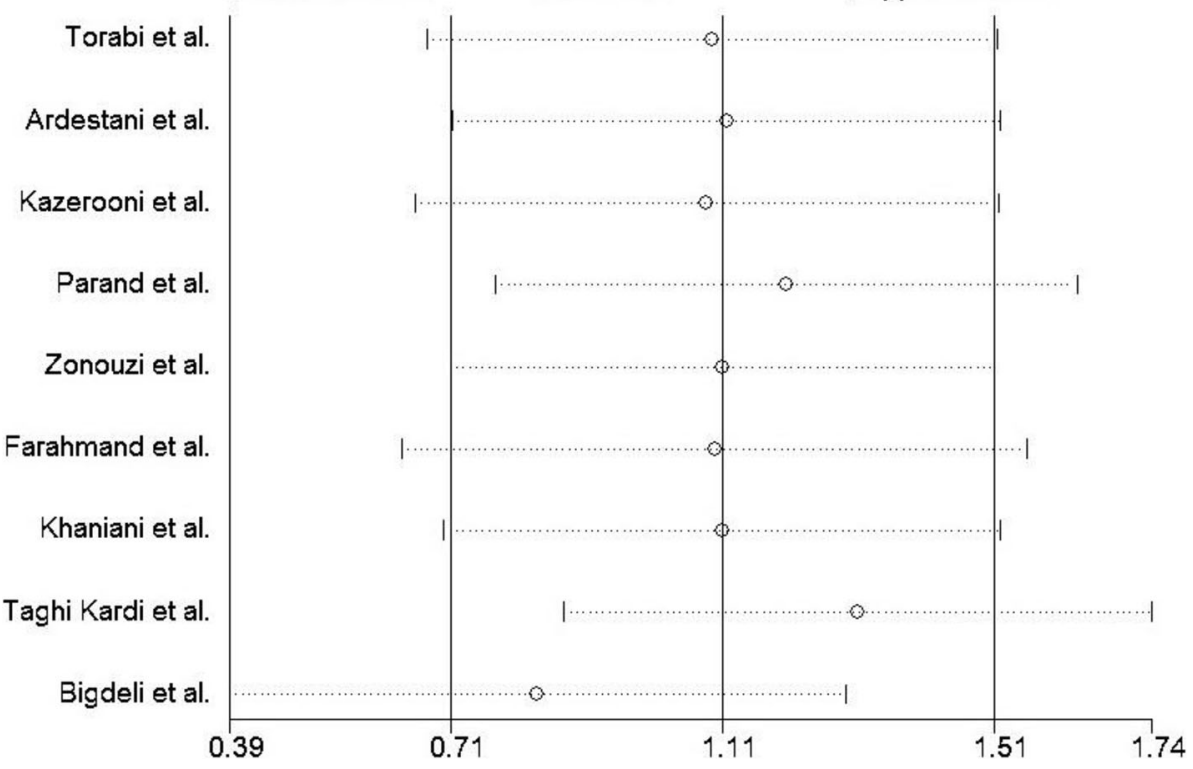

Fig. 6 Sensitivity analysis in the present meta-analysis investigates the association of FVL 1691G > A mutation an risk of RPL; a: overall population, b: Iranian studies

observed heterogeneity expect Asians and studies with cohort design. Furthermore, the other way of dealing with statistical heterogeneity, which we used in our analysis, was to incorporate "Random" term to account for it in a random-effects. Random effect model typically produces more conservative estimates of the significance of a result (a wider confidence interval). As it gives proportionately higher weights to smaller studies and lower weights to larger studies than fixed effect analysis.
To address the limitations in the current metaanalysis, it should be stated that, first our literature search was limited to only studies published in English language. Second, there was a degree of heterogeneity during the overall analysis. But not in all subgroup analyses, indicating the role of genetic diversity and other confounders in susceptibility to RPL. Third, as this meta-analysis a crude estimation of the association between FVL 1691G > A mutation and the risk of RPL, 
thus the roles of age, paternal genetic impression, environmental factors, and the effect of gene-gene interactions in conferring the susceptibility risk to RPL were neglected.

Considering all the facts, this meta-analysis, the first one of its type to our best knowledge, retrieved 62 studies, encompassing 10,410 cases and 9406 health controls, to find a consistent result of the association between FVL 1691G > A mutation and risk of RPL. Our results indicated statistically significant increased risk of RPL in the overall analysis. The increased susceptibility to RPL was also observed in Iranian, Asian, European, Africa populations, and studies with case-control design, but not in South Americans and studies with cohort design. Further experiments, alongside with inclusion of additional studies with large sample sizes, should consider the role cofounders in susceptibility to RPL.

\section{Abbreviations}

FVL: Factor V leiden; RPL: Recurrent pregnancy loss;

MTHFR: Methylenetetrahydrofolate reductase; Cl: Confidence interval; OR: Odds ratio; SNP: Single-nucleotide polymorphism; PRISMA: Preferred reporting items for systematic reviews and meta-analyses; NOS: NewcastleOttawa scale; HWE: Hardy-Weinberg equilibrium

\section{Acknowledgments}

We would like to thank Dr. Saeed Aslani for his valuable comments that greatly improved the manuscript.

\section{Authors' contributions}

ME and BR originated the study, acquired data. BR and MK performed statistical analysis, interpreted data, drafted the manuscript. SA revised the manuscript. SA and MS approved the manuscript. All authors read and approved the final manuscript.

\section{Funding}

Not applicable.

\section{Availability of data and materials}

All data that support the conclusions of this manuscript are included within the article.

\section{Ethics approval and consent to participate}

Not applicable.

\section{Consent for publication}

Not applicable.

\section{Competing interests}

The authors declare that they have no competing interests.

\section{Author details \\ ${ }^{1}$ Department of Hematology, Faculty of Medical Sciences, Tarbiat Modares University, North Kargar Av, Tehran 14117, Iran. ${ }^{2}$ Department of Basic sciences, Maragheh University of medical sciences, Maragheh, Iran. ${ }^{3}$ Rahat Breach and Sleep Research Center, Tabriz University of Medical Sciences, Tabriz, Iran.}

Received: 12 January 2020 Accepted: 20 May 2020

Published online: 24 June 2020

\section{References}

1. Reddy UM. Management of pregnancy after stillbirth. Clin Obstet Gynecol. 2010;53(3):700-9.

2. Ford HB, Schust DJ. Recurrent pregnancy loss: etiology, diagnosis, and therapy. Rev Obstet Gynecol. 2009;2(2):76.
3. von Eye Corleta H. It is time to respect the American Society for Reproductive Medicine definition of recurrent pregnancy loss. Fertil Steril. 2010;94(4):e61.

4. Rai R, Regan L. Recurrent miscarriage. Lancet. 2006;368(9535):601-11.

5. Li T, et al. Recurrent miscarriage: aetiology, management and prognosis. Hum Reprod Update. 2002;8(5):463-81.

6. Allison $J \mathrm{~L}$, Schust DJ. Recurrent first trimester pregnancy loss: revised definitions and novel causes. Curr Opin Endocrinol, Diabetes Obes. 2009; 16(6):446-50.

7. McNamee K, Dawood F, Farquharson R. Recurrent miscarriage and thrombophilia: an update. Curr Opin Obstet Gynecol. 2012;24(4):229-34.

8. Obstetricians, A.C.o. and Gynecologists, Frequently asked questions 100, pregnancy: repeated miscarriages [Internet]. Washington, DC: The American College of Obstetricians and Gynecologists; c2017 [cited 2017 Oct 25].

9. Brenner B. Haemostatic changes in pregnancy. Thromb Res. 2004;114(5-6): 409-14.

10. Uchikova EH, Ledjev II. Changes in haemostasis during normal pregnancy. Eur J Obstet Gynecol Reprod Biol. 2005;119(2):185-8.

11. Buchholz T, Thaler CJ. Inherited thrombophilia: impact on human reproduction. Am J Reprod Immunol. 2003;50(1):20-32

12. Di Micco $P$, et al. Recurrent pregnancy loss and thrombophilia. Clin Lab. 2007;53(5/6):309

13. Coulam CB, et al. Multiple thrombophilic gene mutations rather than specific gene mutations are risk factors for recurrent miscarriage. Am J Reprod Immunol. 2006:55(5):360-8.

14. Foka Z, et al. Factor V Leiden and prothrombin G20210A mutations, but not methylenetetrahydrofolate reductase C677T, are associated with recurrent miscarriages. Hum Reprod. 2000;15(2):458-62.

15. Grandone $E_{\text {, et }}$ al. Factor $V$ Leiden is associated with repeated and recurrent unexplained fetal losses. Thromb Haemost. 1997;77(05):0822-4.

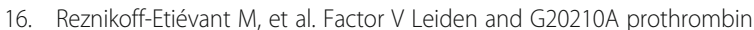
mutations are risk factors for very early recurrent miscarriage. Br J Obstet Gynaecol. 2001;108(12):1251-4.

17. Serrano F, et al. Factor V Leiden and prothrombin G20210A in Portuguese women with recurrent miscarriage: is it worthwhile to investigate? Arch Gynecol Obstet. 2011;284(5):1127-32.

18. Kujovich JL. Factor $v$ Leiden thrombophilia. Genetics in Medicine. 2011;13(1):

19. Lindqvist PG, et al. Activated protein C resistance (FV: Q506) and pregnancy. Thromb Haemost. 1999:81(04):532-7.

20. Svensson PJ, Dahlback B. Resistance to activated protein C as a basis for venous thrombosis. N Engl J Med. 1994;330(8):517-22.

21. Mohammadi, M.M.A., M.G. Al-Halabi, and F.M.S. Monem, Prevalence of factor $\checkmark$ Leiden mutation and its relation with recurrent spontaneous pregnancy loss in a group of Syrian women. 2007.

22. Rodeghiero F, Tosetto A. Activated protein C resistance and factor $V$ Leiden mutation are independent risk factors for venous thromboembolism. Ann Intern Med. 1999:130(8):643-50.

23. Baek K-H, Lee E-J, Kim Y-S. Recurrent pregnancy loss: the key potential mechanisms. Trends Mol Med. 2007:13(7):310-7.

24. Jivraj $S$, et al. Pregnancy outcome in women with factor $V$ Leiden and recurrent miscarriage. BJOG Int J Obstet Gynaecol. 2009;116(7):995-8.

25. Moher $\mathrm{D}$, et al. Preferred reporting items for systematic reviews and metaanalyses: the PRISMA statement. Ann Intern Med. 2009;151(4):264-9.

26. Stang A. Critical evaluation of the Newcastle-Ottawa scale for the assessment of the quality of nonrandomized studies in meta-analyses. Eur J Epidemiol. 2010;25(9):603-5.

27. Huedo-Medina TB, et al. Assessing heterogeneity in meta-analysis: Q statistic or $1^{2}$ index? Psychol Methods. 2006;11(2):193.

28. Mantel N, Haenszel W. Statistical aspects of the analysis of data from retrospective studies of disease. J Natl Cancer Inst. 1959:22(4):719-48.

29. DerSimonian R, Laird N. Meta-analysis in clinical trials. Control Clin Trials. 1986:7(3):177-88.

30. Begg CB, Mazumdar M. Operating characteristics of a rank correlation test for publication bias. Biometrics. 1994:50(4):1088-101.

31. Egger $M$, et al. Bias in meta-analysis detected by a simple, graphical test. Bmj. 1997:315(7109):629-34

32. Dissanayake VH, et al. Candidate gene study of genetic thrombophilic polymorphisms in pre-eclampsia and recurrent pregnancy loss in Sinhalese women. J Obstet Gynaecol Res. 2012;38(9):1168-76. 
33. Pihusch $\mathrm{R}$, et al. Thrombophilic gene mutations and recurrent spontaneous abortion: prothrombin mutation increases the risk in the first trimester. Am J Reprod Immunol. 2001;46(2):124-31.

34. Souza $\mathrm{S}$, et al. Factor $V$ Leiden and factor II G20210A mutations in patients with recurrent abortion. Hum Reprod. 1999;14(10):2448-50.

35. Brenner B, et al. Thrombophilic polymorphisms are common in women with fetal loss without apparent cause. Thromb Haemost. 1999;82(07):6-9.

36. Wramsby M, Sten-Linder M, Bremme K. Primary habitual abortions are associated with high frequency of factor $V$ Leiden mutation. Fertil Steril. 2000;74(5):987-91.

37. Murphy RP, et al. Prospective evaluation of the risk conferred by factor $V$ Leiden and thermolabile methylenetetrahydrofolate reductase polymorphisms in pregnancy. Arterioscler Thromb Vasc Biol. 2000;20(1):26670.

38. Finan RR, et al. Prevalence of factor V G1691A (factor V-Leiden) and prothrombin G20210A gene mutations in a recurrent miscarriage population. Am J Hematol. 2002;71(4):300-5.

39. Pauer HU, et al. Analyzes of three common thrombophilic gene mutations in German women with recurrent abortions. Acta Obstet Gynecol Scand. 2003;82(10):942-7.

40. Mahjoub T, et al. Association between adverse pregnancy outcomes and maternal factor V G1691A (Leiden) and prothrombin G20210A genotypes in women with a history of recurrent idiopathic miscarriages. Am J Hematol. 2005;80(1):12-9.

41. Altintas A, et al. Factor $V$ Leiden and G20210A prothrombin mutations in patients with recurrent pregnancy loss: data from the southeast of Turkey. Ann Hematol. 2007;86(10):727-31.

42. Toth $B$, et al. Paternal thrombophilic gene mutations are not associated with recurrent miscarriage. Am J Reprod Immunol. 2008;60(4):325-32.

43. Biswas $A$, et al. Recurrent abortions in Asian Indians: no role of factor $V$ Leiden Hong Kong/Cambridge mutation and MTHFR polymorphism. Clin Appl Thromb Hemost. 2008;14(1):102-4.

44. Mukhopadhyay R, Saraswathy KN, Ghosh PK. MTHFR C677T and factor V Leiden in recurrent pregnancy loss: a study among an endogamous group in North India. Genet Test Mole Biomark. 2009;13(6):861-5.

45. Ciacci $C$, et al. Early pregnancy loss in celiac women: the role of genetic markers of thrombophilia. Dig Liver Dis. 2009;41(10):717-20.

46. Mohamed MA, et al. Thrombophilic gene mutations in women with repeated spontaneous miscarriage. Genet Test Mole biomark. 2010;14(5): 593-7.

47. Hussein AS, Darwish H, Shelbayeh K. Association between factor V Leiden mutation and poor pregnancy outcomes among Palestinian women. Thromb Res. 2010;126(2):e78-82.

48. Mierla D, et al. Association of prothrombin (A20210G) and factor $V$ Leiden (A506G) with recurrent pregnancy loss. Maedica. 2012;7(3):222.

49. Ozdemir $\mathrm{O}$, et al. Recurrent pregnancy loss and its relation to combined parental thrombophilic gene mutations. Genet Test Mole Biomark. 2012;16(4):279-86.

50. Torabi R, et al. Combination of thrombophilic gene polymorphisms as a cause of increased the risk of recurrent pregnancy loss. J Reprod Infertility. 2012;13(2):89.

51. Cardona $\mathrm{H}$, et al. Lack of association between recurrent pregnancy loss and inherited thrombophilia in a group of Colombian patients. Thrombosis. 2012;2012.

52. Kazerooni T, et al. Correlation between thrombophilia and recurrent pregnancy loss in patients with polycystic ovary syndrome: a comparative study. J Chin Med Assoc. 2013;76(5):282-8.

53. Parand A, et al. Inherited thrombophilia and recurrent pregnancy loss. Iran Red Crescent Med J. 2013;15(12):e13708. (PMID: 24693393).

54. Zonouzi AP, et al. The association between thrombophilic gene mutations and recurrent pregnancy loss. J Assist Reprod Genet. 2013;30(10):1353-9.

55. Pietropolli $A$, et al. Plasminogen activator inhibitor-1, factor $V$, factor $\|$ and methylenetetrahydrofolate reductase polymorphisms in women with recurrent miscarriage. J Obstet Gynaecol. 2014;34(3):229-34.

56. Sharma $A$, et al. Polymorphisms in factor $V$ and antithrombin III gene in recurrent pregnancy loss: a case-control study in Indian population. J Thromb Thrombolysis. 2015;39(4):481-8.

57. Kashif $S$, Kashif MA, Saeed A. The association of factor $V$ Leiden mutation with recurrent pregnancy loss. J Pak Med Assoc. 2015;65(11):1169-72.

58. Gonçalves, R.O., et al., Association between the thrombophilic polymorphisms MTHFR C677T, factor V Leiden, and prothrombin G20210A and recurrent miscarriage in Brazilian women. 2016.
59. Khaniani MS, et al. Evaluation of Thrombophilic genes in recurrent pregnancy loss: a case-control study in Iranian women. Int J Hum Genet. 2016;16(1-2):48-52.

60. Wolski $\mathrm{H}_{\text {, et }}$ al. Contribution of inherited thrombophilia to recurrent miscarriage in the polish population. Ginekol Pol. 2017;88(7):385-92.

61. Elgari MM, et al. Frequency of Thrombophilic gene mutations in patients with deep vein thrombosis and in women with recurrent pregnancy loss. Open Life Sci. 2017;12(1):162-6.

62. Mahmutbegović E, et al. Prevalence of F5 1691G> a, F2 20210G> a, and MTHFR 677 C > T polymorphisms in Bosnian women with pregnancy loss. Bosn J Basic Med Sci. 2017;17(4):309.

63. Bigdeli $R$, et al. Association between thrombophilia gene polymorphisms and recurrent pregnancy loss risk in the Iranian population. Syst Biol Reprod Med. 2018;64(4):274-82.

64. Reddy $R$, et al. Recurrent pregnancy loss: can factor $V$ Leiden mutations be a cause. Obstet Gynecol Sci. 2019;62(3):179-82.

65. Xu Z, et al. Polymorphisms of F2, PROC, PROZ, and F13A1 genes are associated with recurrent spontaneous abortion in Chinese Han women. Clin Appl Thromb Hemost. 2018;24(6):894-900.

66. Kardi MT, et al. Association of factor V Leiden and prothrombin G20210A polymorphisms in women with recurrent pregnancy loss in Isfahan province, Iran. Int J Prev Med. 2018;9(13):8-14. (PMID: 29541428).

67. Badawy A, AlSel BA, Fawzy M. Factor V Leiden G1691A and Prothrombin G20210A mutations are associated with repeated spontaneous miscarriage in Northern area of Saudi Arabia. Genet Mol Res. 2017;16(4):gmr16039810.

68. Farahmand $\mathrm{K}$, et al. Thrombophilic genes alterations as risk factor for recurrent pregnancy loss. J Matern Fetal Neonatal Med. 2016;29(8):1269-73.

69. Ardestani MT, et al. Case control study of the factor $\vee$ Leiden and factor II G20210A mutation frequency in women with recurrent pregnancy loss. Iranian journal of reproductive medicine. 2013;11(1):61.

70. Parveen F, Shukla A, Agrawal S. Should factor V Leiden mutation and prothrombin gene polymorphism testing be done in women with recurrent miscarriage from North India? Arch Gynecol Obstet. 2013;287(2):375-81.

71. Kaur $L$, et al. Genetic thromobophilia in pregnancy: a case-control study among north Indian women. J Thromb Thrombolysis. 2013;35(2):250-6.

72. Ivanov PD, et al. Association of inherited thrombophilia with embryonic and postembryonic recurrent pregnancy loss. Blood Coagul Fibrinolysis. 2009; 20(2):134-40.

73. Sotiriadis A, et al. Combined thrombophilic mutations in women with unexplained recurrent miscarriage. Am J Reprod Immunol. 2007:57(2):133-41.

74. Eroglu Z, et al. Frequency of factor V Leiden (G1691A), prothrombin (G20210A) and methylenetetrahydrofolate reductase (C677T) genes mutations in woman with adverse pregnancy outcome. J Turk German Gynecol Assoc. 2006;7:195-201.

75. Aksoy $M$, et al. The role of thrombofilia related to factor $V$ Leiden and factor II G20210A mutations in recurrent abortions. J Pak Med Assoc. 2005;55(3): 104-8.

76. Hohlagschwandtner $M$, et al. Combined thrombophilic polymorphisms in women with idiopathic recurrent miscarriage. Fertil Steril. 2003;79(5):1141-8.

77. Jusić $A$, et al. The association of factor V G1961A (factor $V$ Leiden), prothrombin G20210A, MTHFR C677T and PAl-1 4G/5G polymorphisms with recurrent pregnancy loss in Bosnian women. Med Glas (Zenica). 2018;15(2): 158-63.

78. Isaoglu U, et al. The association between inherited thrombophilia and recurrent pregnancy loss in Turkish women. Clin Exp Obstet Gynecol. 2014; 41(2):177-81.

79. Baumann $\mathrm{K}$, et al. Maternal factor $\mathrm{V}$ Leiden and prothrombin mutations do not seem to contribute to the occurrence of two or more than two consecutive miscarriages in Caucasian patients. Am J Reprod Immunol. 2013;70(6):518-21.

80. Karata $\mathrm{S}$, et al. Hereditary thrombophilia, anti-beta2 glycoprotein 1 lgM, and anti-annexin $\mathrm{V}$ antibodies in recurrent pregnancy loss. Am J Reprod Immunol. 2012;67(3):251-5.

81. Suer $\mathrm{N}$, et al. Inherited thrombophilia with recurrent pregnancy loss in Turkish women-a real phenomenon? Ginekol Pol. 2012;83(8).

82. Yengel I, Yorulmaz T, Api M. Association between FVL G1691A, FII G20210A and MTHFR C677T and A1298C polymorphisms and Turkish women with recurrent pregnancy loss. Medicinski Glasnik. 2020;17(1).

83. Wingeyer, S.P., et al., Inherited thrombophilia and pregnancy loss. Study of an Argentinian cohort. Medicina Clínica (English Edition), 2019. 152(7): p. 249-254. 
84. Lino FL, et al. Thrombophilic mutations and polymorphisms, alone or in combination, and recurrent spontaneous abortion. Clin Appl Thromb Hemost. 2015;21(4):365-72.

85. Dutra CG, et al. Lack of association between thrombophilic gene variants and recurrent pregnancy loss. Hum Fertil. 2014;17(2):99-105.

86. Mtiraoui $\mathrm{N}$, et al. Prevalence of antiphospholipid antibodies, factor $\mathrm{V}$ G1691A (Leiden) and prothrombin G20210A mutations in early and late recurrent pregnancy loss. Eur J Obstet Gynecol Reprod Biol. 2005;1 19(2): 164-70.

87. Settin $\mathrm{A}$, et al. Factor $\mathrm{V}$ Leiden and prothrombin gene mutations in Egyptian cases with unexplained recurrent pregnancy loss. Hematology. 2011;16(1):59-63.

88. Page JM, Silver RM. Genetic causes of recurrent pregnancy loss. Clin Obstet Gynecol. 2016;59(3):498-508.

89. Rosendaal F, et al. High risk of thrombosis in patients homozygous for factor V Leiden (activated protein C resistance)[see comments]. Blood. 1995; 85(6):1504-8.

90. Ridker PM, et al. Mutation in the gene coding for coagulation factor $V$ and the risk of myocardial infarction, stroke, and venous thrombosis in apparently healthy men. N Engl J Med. 1995;332(14):912-7.

91. Sergi C, Al Jishi T, Walker M. Factor V Leiden mutation in women with early recurrent pregnancy loss: a meta-analysis and systematic review of the causal association. Arch Gynecol Obstet. 2015;291(3):671-9.

92. Cavalcante MB, et al. Coagulation biomarkers in women with recurrent miscarriage and polycystic ovarian syndrome: systematic review and metaanalysis. Geburtshilfe Frauenheilkd. 2019;79(07):697-704.

93. Shi X, et al. Maternal genetic polymorphisms and unexplained recurrent miscarriage: a systematic review and meta-analysis. Clin Genet. 2017;91(2): 265-84

94. Kamali M, et al. Association between thrombophilic genes polymorphisms and recurrent pregnancy loss susceptibility in the Iranian population: a systematic review and meta-analysis. Iran Biomed J. 2018;22(2):78.

\section{Publisher's Note}

Springer Nature remains neutral with regard to jurisdictional claims in published maps and institutional affiliations.

Ready to submit your research? Choose BMC and benefit from:

- fast, convenient online submission

- thorough peer review by experienced researchers in your field

- rapid publication on acceptance

- support for research data, including large and complex data types

- gold Open Access which fosters wider collaboration and increased citations

- maximum visibility for your research: over $100 \mathrm{M}$ website views per year

At $\mathrm{BMC}$, research is always in progress.

Learn more biomedcentral.com/submissions 